\title{
Parallelisms of quadric sets
}

\author{
William E. Cherowitzo Norman L. Johnson
}

\begin{abstract}
In this article, it is shown that every flock of a hyperbolic quadric $H$ and every flock of a quadratic cone $C$ in $\mathrm{PG}(3, K)$, for $K$ a field, is in a transitive parallelism of $H$ or $C$, respectively. Furthermore, it is shown it is possible to have parallelisms of quadratic cones by maximal partial flocks. The theory of parallelisms of quadratic cones is generalized to analogous results for parallelisms of $\alpha$-cones.
\end{abstract}

Keywords: flocks, flokki, parallelisms, hyperbolic quadric, elliptic quadric, quadratic cone, $\alpha$-cone

MSC 2000: 51E20, 51A15

\section{Introduction}

Let $K$ be a field and consider a flock of a quadratic cone, an elliptic quadric or a hyperbolic quadric in $\mathrm{PG}(3, K)$. This article considers whether there are "parallelisms" of these quadric sets, which in each case means a set of mutually disjoint flocks, whose union is a complete cover of the set of conics of plane intersections of the quadric set in question.

Consider first a "hyperbolic parallelism" as a union of mutually disjoint hyperbolic flocks, whose union is the set of all conics that are sections of a hyperbolic quadric.

When $K$ is isomorphic to $\mathrm{GF}(q)$, there are $\left(q^{4}-1\right) /(q-1)$ planes in $\mathrm{PG}(3, q)$, and there are $2(q+1)$ lines that lie in the hyperbolic quadric. Each of these lines lies in $q+1$ planes, none of which can be associated with a hyperbolic flock and each plane that contains a line of a ruling also contains a line of the opposite ruling. Hence, there are $q^{3}+q^{2}+q+1-(q+1)^{2}=q\left(q^{2}-1\right)$ planes that intersect a hyperbolic quadric in a conic. Since a hyperbolic flock contains $q+1$ planes, we would need $q(q-1)$ hyperbolic flocks in a finite hyperbolic parallelism. 





\section{Parallelisms of hyperbolic quadrics}

The reader is again directed to Johnson [8] for background on the translation planes corresponding to flocks of hyperbolic quadrics.

The feature that connects flocks of hyperbolic quadrics with the associated spreads in $\mathrm{PG}(3, q)$ is that they all admit a regulus-inducing affine homology group of order $q-1$. More generally, over an arbitrary field $K$, the associated spreads admit a regulus-inducing affine homology group that fixes two components of some regulus and acts regularly on the remaining components.

Let $F$ be a flock of the hyperbolic quadric $x_{0} x_{3}=x_{1} x_{2}$ in $\mathrm{PG}(3, K)$, whose points are represented by homogeneous coordinates $\left(x_{0}, x_{1}, x_{2}, x_{3}\right)$ where $K$ is a field. Then the set of planes which contain the conics in $F$ may be represented as follows:

$$
\begin{aligned}
\rho & : x_{1}=x_{2}, \\
\pi_{t} & : x_{0}-t x_{1}+f(t) x_{2}-g(t) x_{3}=0
\end{aligned}
$$

for all $t$ in $K$ where $f$ and $g$ are functions of $K$ such that $f$ is bijective. We first point out that there is a natural collineation group of the hyperbolic quadric

$$
G=\left\langle\tau_{a, b}=\left[\begin{array}{cccc}
1 & b & 0 & 0 \\
0 & a & 0 & 0 \\
0 & 0 & 1 & b \\
0 & 0 & 1 & a
\end{array}\right] ; a \neq 0, b \in K\right\rangle
$$

Note that a point $\left(x_{0}, x_{1}, x_{2}, x_{3}\right)$ maps to $\left(x_{0}, x_{1} a+x_{0} b, x_{2}, x_{3} a+x_{2} b\right)$ so if the point is on the hyperbolic quadric then $x_{0} x_{3}=x_{1} x_{2}$, and the image point is on the hyperbolic quadric if and only if $x_{0}\left(x_{3} a+x_{2} b\right)=\left(x_{1} a+x_{0} b\right) x_{2}$, which is clearly valid. In the finite case, we note that $G$ is a group of order $q(q-1)$, which if the hyperbolic quadric is considered a regulus net, would fix a Baer subplane of this regulus net pointwise. Therefore, $\tau_{a, b}$ will map the flock $F$ onto another flock $F \tau_{a, b}$ and $\left\{F \tau_{a, b} ; \tau_{a, b} \in G\right\}$ is a parallelism of the hyperbolic quadric if and only if $F$ and $F \tau_{a, b}$ share no plane. Now the Baer subplane $\Sigma_{0}$ fixed pointwise by $G$ is a ruling line of the hyperbolic quadric and hence each plane of the flock intersects $\Sigma_{0}$ in exactly one point. Now consider any plane $\eta$ of $F$ and assume that $\eta$ contains the point $P$ of $\Sigma_{0}$, then the image of $\eta$ also contains the point $P$, which means that $\eta \tau_{a, b}$ cannot belong to $F$, unless $\eta \tau_{a, b}=\eta$.

The plane $\rho \tau_{a, b}$ is generated as follows: $\langle(1, b, 0,0),(0, a, 1, a),(0,0,0,1)\rangle$, which is clearly not $\pi_{t}$, for any $t$, and is $\rho$ if and only if $a=1$ and $b=0$, so that $\tau_{1,0}$ is the identity mapping. A basis for $\pi_{t}$ is

$$
\{(-f(t), 0,1,0),(t, 1,0,0),(g(t), 0,0,1)\},
$$

which maps under $\tau_{a, b}$ to

$$
\{(-f(t),-f(t) b, 1, b),(t, t b+a, 0,0),(g(t), g(t) b, 0, a)\} .
$$





\section{Parallelisms with maximal partial flocks}

Is it possible to have a parallelism of maximal partial spreads? This question was asked to the second author by Arrigo Bonisoli in the context of parallelisms of the lines of $\mathrm{PG}(3, q)$ and his initial response was that the answer was no! But, ask the question more generally: Is it possible to have a parallelism of the blocks of a geometry by flocks? In particular, is it possible to have a parallelism of the conics of intersection of a quadratic cone in $\mathrm{PG}(3, K)$ by maximal partial flocks? The very surprising answer is, "yes", at least when $K$ is an infinite field.

Consider a putative conical (partial) spread

$$
x=0, y=x\left[\begin{array}{cc}
u & \gamma t^{3} \\
t & u
\end{array}\right] ; t, u \in K, \gamma \text { a non-square in } K \text {. }
$$

The corresponding flock would require that the functions

$$
\phi_{u}: t \mapsto t u^{2}-\left(f(t)=\gamma t^{3}\right)
$$

are bijective for all $u \in K$, in order that we indeed obtain a flock of a quadratic cone. Assume that some function is injective but not bijective. Then we would obtain a partial flock which does not cover all of the points of the quadratic cone, so could not be considered a flock. However, the partial spread listed above is clearly maximal since otherwise there would be a 2-dimensional $K$-subspace $y=x T$, such that $\left[\begin{array}{cc}u & \gamma t^{3} \\ t & u\end{array}\right]-T$ is a non-singular matrix for all $u, t \in K$, clearly a contradiction. So, the question is, when is $\phi_{u}$ injective but not bijective?

We have $t u^{2}-\gamma t^{3}=s u^{2}-\gamma s^{3}$ if and only if for $u \neq 0$, we have $u^{2}=$ $\gamma\left(t^{3}-s^{3}\right) /(t-s)$ if and only if $\gamma\left(t^{2}+s t+s^{2}\right)=u^{2}$ and for $u=0$, we must have $(t / s)^{3}=1$, implies that $t / s=1$. We note that $u^{2}-\gamma t^{4}=0$, would imply that $\gamma$ is a square. Hence, consider

$$
\left(t^{2}+s t+s^{2}\right)=u^{2} / \gamma
$$

as a quadratic in $t$. The discriminant is $s^{2}-4\left(s^{2}-u^{2} / \gamma\right)=-3 s^{2}+4 u^{2} / \gamma$. Now let $K$ be an ordered field and let $\gamma$ be any negative element of $K$. Then the discriminant is negative so can never be square. Therefore, $f(t)=\gamma t^{3}$ is injective in any ordered field such that $v^{3}=1$, implies $v=1$. But $v^{3}=1$, and $v$ not 1 means that $v^{2}+v+1=0$, which has discriminant $1-4=-3$. Hence, the question is then whether in an ordered field when the function $f(t)=\gamma t^{3}$ is injective is it also bijective? Let $K$ be a subfield of the field of real numbers that does not contain all cube roots of each of its elements. Then, $t^{3}=\delta$, for $\delta$ in $K$, where $\sqrt[3]{\delta}$ is not in $K$, is not surjective. For example, the rational field will have this property as well as infinitely many subfields of the field of real numbers. 
Theorem 4.1. Let $K$ be an ordered field such that the function $t \mapsto \gamma t^{3}$ is injective but not bijective, where $\gamma$ is a fixed negative element in $K$. Then there is a maximal partial flock $\mathcal{F}$ of a quadratic cone in $\mathrm{PG}(3, K)$ with planes

$$
t x_{0}-\gamma t^{3} x_{1}+x_{3}=0, t \in K
$$

Now we apply the argument of Theorem 2.2 with the group

$$
G=\left\langle\tau_{a, b}=\left[\begin{array}{cc}
I & {\left[\begin{array}{cc}
a & b \\
0 & 0
\end{array}\right]} \\
0 & I
\end{array}\right] ; a, b \in K\right\rangle,
$$

to obtain a set $\mathcal{F} G$ of mutually disjoint flocks, where

$$
\mathcal{F}_{a, b}: t x_{0}-\left(\gamma t^{3}+a\right) x_{1}+b x_{2}+x_{3}=0 .
$$

Since the set of all planes of intersection have the form $c x_{0}+d x_{1}+e x_{2}+x_{3}=0$, the set of flocks clearly cover all planes that do not contain a generator line of the cone and hence cover all conics of intersection.

Definition 4.2. For a field $K$, assume that there are functions $f(t)$ and $g(t)$ on $K$ so that the functions $\phi_{u}: t \mapsto t u^{2}-f(t)+g(t) u$ are all injective but not all bijective on $K$. We then say that corresponding maximal partial flock is "injective and not bijective".

Thus, we have the following theorem.

Theorem 4.3. Let $\mathcal{F}$ be an injective and not bijective maximal partial flock of a quadratic cone in $\mathrm{PG}(3, K)$. Then there is a transitive parallelism of the quadratic cone (conics of intersection) by maximal partial flocks.

\section{Parallelisms of $\alpha$-cones}

Recently in [6], the authors consider a generalization of quadratic cones, which we shall call $\alpha$-cones in this article. We consider here when there are parallelisms of $\alpha$-cones by $\alpha$-flokki.

Let $\alpha$ be an automorphism of a field $K$ and consider PG $(3, K)$, represented in the form $\left(x_{0}, x_{1}, x_{2}, x_{3}\right) ; x_{i} \in K$, as homogeneous coordinates. Let an $\alpha$-cone $\mathcal{C}_{\alpha}$ be defined by $x_{0} x_{1}^{\alpha}=x_{2}^{\alpha+1}$, with vertex $(0,0,0,1)$. The intersection of a plane not through the vertex and an $\alpha$-cone is called an " $\alpha$-conic". This terminology is adopted in analogy with quadratic cones, but one should be aware that $\alpha$-conics are in general not even ovals. 



